\title{
GEOARQUEOLOGIA EN LA LOCALIDAD NUTRIA MANSA (PDOS. DE GRAL. ALVARADO Y LOBERIA, PROVINCIA DE BUENOS AIRES)
}

\author{
Cristian M. Favier-Dubois ${ }^{1}$ y Mariano Bonomo ${ }^{2}$
}

1-CONICET-INCUAPA, Facultad de Ciencias Sociales, UNCPBA. cfavier@coopenet.com.ar

2-CONICET, Facultad de Ciencias Naturales y Museo, UNLP.mbonomo@fcnym.unlp.edu.ar

Presentado el: 25/11/2007 - Aceptado 02/04/2008

\section{Resumen}

En este trabajo se presentan estudios geoarqueológicos realizados en la localidad arqueológica Nutria Mansa, localizada en las márgenes del arroyo homónimo en las proximidades del litoral marítimo bonaerense. Las investigaciones arqueológicas comenzaron en el año 2000 habiéndose reconocido dos sitios en superficie (NM1sup y NM2sup), cuyos materiales fueron recuperados en zonas afectadas por el arado, y un sitio en estratigrafía (NM1), en el que se excavó una superficie de 23 $m^{2}$ recuperándose material lítico y muy abundantes restos faunísticos (unas 140.000 piezas) correspondientes al Holoceno tardío. Los análisis geoarqueológicos estuvieron orientados a profundizar aspectos estratigráficos, así como a evaluar los procesos de formación y entorno ambiental de la evidencia arqueológica recuperada en la localidad. Los resultados obtenidos han permitido explicar algunos de los procesos tafonómicos involucrados en el sitio excavado, así como generar expectativas arqueológicas para diferentes posiciones del paisaje ocupado por las poblaciones prehispánicas: divisorias loéssicas (suelos naturales y arados), pendientes y valle, contribuyendo al análisis de la resolución, integridad y preservación de la evidencia antrópica en cada sector.

Palabras clave: Geoarqueología, Arroyo Nutria Mansa, Holoceno tardio.

\begin{abstract}
Geoarchaeological studies carried out in the Nutria Mansa archaeological locality (Southern Buenos Aires Province) are presented. Archaeological research started in this area in 2000, revealing the presence of two surface sites (NM1sup y NM2sup) where artifacts were exposed by plowing, and one site in stratigraphy (NM1) where a $23 \mathrm{~m}^{2}$ excavation provided lithic material and very abundant faunal remains (about 140.000 pieces). The geoarchaeological analyses were focused in stratigraphy, and the evaluation of formation processes and environmental context of the archaeological record found at the Nutria Mansa locality. Among the results of this work are the explanation of some taphonomic processes involved in the excavated site NM1, and the generation of expectations about integrity, resolution and preservation of the cultural evidence in different places of the landscape occupied by Prehispanic people as the loessic divide (no disturbed and plowed soils), slopes, and the main valley.
\end{abstract}

Key words: Geoarchaeology, Nutria Mansa stream, Late Holocene. 


\section{Introducción}

Desde los comienzos de las investigaciones sobre las poblaciones prehispánicas pampeanas distintos autores (Ameghino 1880; Bórmida 1960; Frenguelli y Outes 1924; Hrdlicka 1912; Menghín y Bórmida 1950; Tapia 1937) han utilizado información geológica para interpretar los contextos arqueológicos. Particularmente, intentaban correlacionar los artefactos hallados con la edad estimada para las unidades estratigráficas que los contenían y en menor medida explicar procesos naturales involucrados en la formación de los depósitos arqueológicos. A mediados de la década de 1960 y principios de la de 1970, Madrazo y su equipo de colaboradores interactuaron de manera sostenida con geólogos y paleontólogos (Fidalgo et al. 1971; Madrazo 1972, 1979; Teruggi 1968; Zetti et al. 1972). Con estos estudios se ampliaron los temas abordados desde la óptica geológica, incluyendo análisis de muestras sedimentológicas para establecer las secuencias estratigráficas y cronológicas relativas de los sitios, la vinculación de estos perfiles con los marcos geológicos regionales y las oscilaciones climáticas, la génesis de reparos rocosos con ocupaciones humanas y la determinación de las rocas empleadas como materia prima para confeccionar artefactos.

Siguiendo las bases delineadas en los trabajos previos, desde la década de 1980 en adelante se consolida un modo multidisciplinario de estudiar el pasado pampeano con la participación formal de especialistas del campo de la geología del Cuaternario en las investigaciones arqueológicas (véase Zarate y Prieto 1997). Se trataron temas variados como la cronología, esquemas estratigráficos del Pleistoceno final-Holoceno, contextos sedimentológicos y geomorfológicos, los procesos de formación de los sitios, la composición mineralógica y química de materiales líticos y cerámicos, las potenciales áreas de aprovisionamiento de rocas y arcillas, la influencia de los cambios climáticos en la historia ocupacional de la región, etc. (Fidalgo et al. 1986; Flegenheimer y Zárate 1993, Flegenheimer et al. 1999; González y Weiler 1988; González de Bonaveri y Zárate 1993-94; Martínez y Osterrieth 2001; Messineo et al. 2004; Osterrieth et al. 2002; Pérez Meroni y Blasi 1997; Politis 1984; Zárate et al. 20002002; Zavala et al. 1992, entre otros). Dentro de este período se destacan aquellos que adoptaron explícitamente una perspectiva geoarqueológica e integraron distintas escalas espaciales de análisis, como los estudios efectuados en las localidades Cerro La China (Zárate y Flegenheimer 1991), Paso Otero (Favier Dubois 2006; Holliday et al. 2003), Zanjón Seco (Favier Dubois y Politis 2006), La Guillerma (González 2005) y en la costa central (Aldazábal et al. 2004).

Si bien con algunos matices de acuerdo a la aproximación, la geoarqueología puede considerarse investigación arqueológica utilizando métodos y conceptos de las Ciencias de la Tierra (Butzer 1982), superando las dicotomías de escalas e intereses que tradicionalmente permeaban el trabajo interdisciplinario a partir de una perspectiva centrada en los agentes humanos y su interacción con el paisaje. En este artículo se presentan los estudios geoarqueológicos efectuados en la localidad arqueológica Nutria Mansa (Bonomo 2005). Los mismos estuvieron orientados a profundizar aspectos estratigráficos, así como a evaluar el contexto geoambiental de la evidencia arqueológica recuperada en la localidad (Favier Dubois y Bonomo 2002). Los resultados obtenidos han permitido explicar parte de los procesos tafonómicos involucrados en el sitio NM1, y generar expectativas arqueológicas en diferentes posiciones del paisaje local: divisorias loéssicas (suelos naturales y arados), pendientes, y valle fluvial, contribuyendo al análisis de la resolución, integridad y preservación de la evidencia antrópica en cada caso. 


\section{Marco geológico regional}

En relación con el marco geológico, afloran en este sector unidades de carácter regional correspondientes al Pleistoceno tardío y Holoceno. Se trata de sedimentos fluviales y lacustres referidos a los Miembros Guerrero y Río Salado de la Formación Luján ("Lujanense" y "Platense" en la terminología de Ameghino y Frenguelli), a los que suprayacen aquellos de origen eólico de la Formación La Postrera (Fidalgo et al. 1973). Estas unidades apoyan sobre la Formación Pampiano (parte de los "sedimentos pampeanos" de la terminología clásica), constituida principalmente por loess, la cual tiene una gran extensión y forma un sustrato con leves ondulaciones que caracteriza el paisaje de la región.

El Miembro Guerrero de la Formación Luján está compuesto por depósitos fluviales de planicie de inundación, constituyéndose en la evidencia más temprana del comienzo del desarrollo de la red de drenaje actual. Los sedimentos de la base están integrados por arenas finas y limos con coloraciones castañas y los de la parte superior están constituidos por arenas limosas y limos arcillosos arenosos de color verde amarillento. Para el sector verde de este miembro se obtuvieron dataciones radiocarbónicas que comprenden desde los 28.600 a los 10.000 años A.P. (Bonadonna et al. 1995, Tonni y Cione 1995; Tonni et al. 1999, Tonni et al. 2003). En algunos sectores, sobre la parte superior del Miembro Guerrero, puede registrarse una unidad edafoestratigráfica de coloración oscura denominada Suelo Puesto Callejón Viejo que representaría el límite Pleistoceno-Holoceno, fechado entre los 10.000 y 9.000 años A.P. (Bonadonna et al. 1995, Johnson et al. 1998, Zárate et al. 1998, Martínez 2001).

Por encima del Miembro Guerrero, o sobre los remanentes del paleosuelo Puesto Callejón Viejo, se localiza el Miembro Río Salado de la Formación Luján, depósito de ambiente palustrelacustre y en menor medida fluvial. Este miembro posee tonalidades grises a blanquecinas y está constituido por limos arenosos a arcillosos con cantidades variables de materia orgánica y abundante contenido bioclástico. Para estos depósitos se obtuvieron dataciones que abarcan desde los 10.800 a los 3.400 años A.P. (Bonadonna et al. 1995). En la parte superior del Miembro RíoSalado, se presentan en forma esporádica remanentes del Suelo Puesto Berrondo, rico en materia orgánica, que fue datado entre 3.000 y 2.000 años A.P. (Tonni et al. 2001).

La Fm. La Postrera, de origen eólico, posee una amplia distribución en la Pampa Húmeda. Comienza a sedimentarse sobre las divisorias, contemporáneamente con la parte superior del Miembro Guerrero, hasta épocas recientes (Fidalgo et al. 1973). Durante el Holoceno tardío, su parte superior también está representada en los cursos fluviales. Se halla constituida por sedimentos sin consolidar, conformados por limos arenosos y arenas limosas. Estas acumulaciones poseen un color castaño amarillento uniforme, aspecto homogéneo y ausencia de estratificación. Esta unidad puede dividirse en una parte inferior, con una cronología que va desde el Pleistoceno final hasta los 8.300 años A.P. (Tonni 1994; Tonni y Cione 1995), separada por una discordancia erosiva de una parte superior, con espesor variable y edades que se extienden hasta los 440 años A.P. (Tonni et al. 1999).

\section{Localidad arqueológica Nutria Mansa}

La localidad arqueológica Nutria Mansa se ubica a ambas márgenes del arroyo homónimo, en las proximidades del poblado de Centinela del Mar, a 3,5 km del litoral marítimo bonaerense (Figura 1). Los estudios arqueológicos se iniciaron en el año 2000 habiéndose reconocido hasta 
el momento dos sitios en superficie: NM1sup y NM2sup, cuyos materiales fueron recuperados en zonas afectadas por el laboreo agrícola y un sitio en estratigrafía: NM1, en el que se excavó una superficie de $23 \mathrm{~m}^{2}$ (Bonomo 2005).

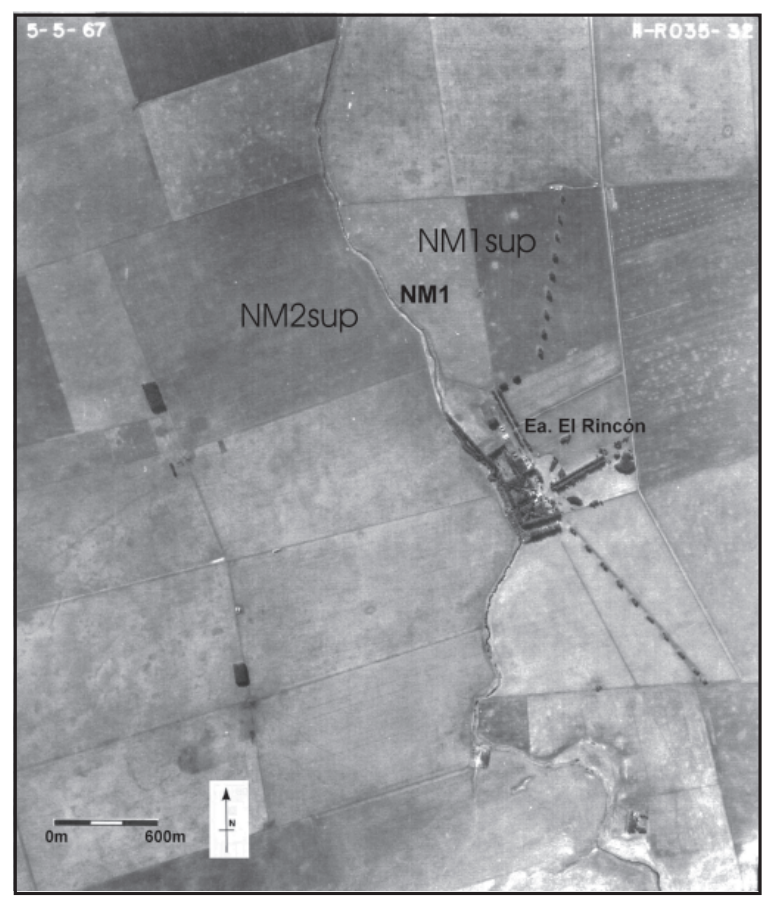

Figura 1. Vista Aérea

El sitio NM1sup se ubica en el potrero no 6 de la Ea. El Rincón en el partido de Gral. Alvarado, adyacente al sitio en posición estratigráfica Nutria Mansa 1 (Figura 1). Allí fueron recuperados 123 artefactos líticos, 8 rodados costeros sin modificación antrópica, 2 fragmentos de bivalvos marinos, escasos fragmentos óseos de mamíferos indeterminados, restos de vidrio y loza, así como varios elementos de plástico.

El sitio NM2sup se encuentra localizado en las Eas. Nutria Mansa y La Maruja en el partido de Lobería (Figura 1). En este lugar se recuperaron 867 artefactos líticos, 3 fragmentos de pigmento mineral, 3 tiestos de alfarería lisa, 1 fragmento rodado de molusco marino y 86 rodados costeros sin modificar con longitudes máximas que varían entre 7,5 y $2,5 \mathrm{~cm}$. También fueron hallados escasos fragmentos óseos indeterminados, algunos de ellos carbonizados o calcinados, fragmentos de vidrio, loza, ladrillo y varios rodados muy pequeños (menores a 1 $\mathrm{cm}$ ) de materias primas semejantes a las costeras.

En ambos sitios superficiales se registró una gran diversidad de artefactos líticos. Entre ellos, se incluyen numerosos instrumentos de molienda (morteros, molinos, manos) y núcleos de cuarcita con un gran potencial de materia prima sin aprovechar, junto a alfarería, pigmentos minerales y significativas proporciones de instrumentos manufacturados mediante lascados, los cuales señalan el desarrollo de múltiples actividades. 
Por su parte, el sitio en estratigrafía NM1 posee dos unidades arqueológicas discretas. El Componente Inferior posee una amplia distribución que abarca desde los 75 hasta los 160 $\mathrm{cm}$ desde el nivel 0 . En este componente se recuperó la notable cantidad de 142.732 restos faunísticos, 2.292 artefactos líticos, 13 pigmentos minerales y 77 ecofactos.

A través de los estudios de tecnología lítica efectuados con los materiales del Componente Inferior de NM1 se infiere el desarrollo de una gran variedad de tareas de producción artefactual mediante la utilización de materias primas procedentes del Sistema Serrano de Tandilia y de rodados de la costa atlántica. En el sitio se redujeron núcleos para la obtención de lascas que fueron transformadas en diversos instrumentos con filos retocados. Con relación al material faunístico, los estudios del conjunto muestran que la especie más abundante es el guanaco (Lama guanicoe) que posee un número mínimo de 58 individuos, determinado a partir de mandíbulas y dientes aislados (Bonomo et al. 2005). En el sitio está representada asimismo una gran diversidad de mamíferos correspondientes a ambientes áridos y semiáridos (Reithrodon auritus, Zaedyus pichiy, Dolichotis patagonum, Dusicyon avus y Lama guanicoe), templados y húmedos (Dusicyon gymnocercus y Dasypus hybridus) y subtropicales (Myocastor coipus y Chrysocyon brachyurus). Las evidencias registradas en NM1 sugieren el desarrollo de múltiples actividades en un campamento en el que se realizaron tareas de procesamiento y consumo de un amplio rango de partes esqueletarias de guanaco (Bonomo 2005).

\section{Metodología}

Se realizó un análisis de la estratigrafía a escala puntual y local (loci arqueológicos y áreas adyacentes a los mismos) de acuerdo con la metodología propuesta por Butzer (1982). Se articuló la estratigrafía de estas secuencias con aquellas de mayor escala (geología regional), intentando caracterizar los ambientes representados en el sector en diferentes momentos.

Para la descripción de los perfiles se utilizó una perspectiva pedoestratigráfica antes que la litoestratigráfica utilizada en trabajos previos en la región (p. ej. Fidalgo et al. 1971, 1986; Zetti et al. 1972), ya que permite una calibración más adecuada de la representación temporal de las secuencias en estudio y una mejor caracterización de los procesos postdepositacionales involucrados en la formación de los depósitos arqueológicos (Holliday et al. 1993). Con respecto a las técnicas de laboratorio utilizadas, se determinó textura por tamizado y pipeteo; color en seco de acuerdo a la Tabla Munsell (Munsell Soil Color Charts); presencia de $\mathrm{CO}_{3} \mathrm{Ca}$ por su reacción con $\mathrm{HCl}$; porcentaje de materia orgánica por el método Walkley-Black; y $\mathrm{pH}$ en pasta (1:2.5). Asimismo, se determinaron las bases presentes y la salinidad de los sedimentos. Todos estos análisis fueron efectuados en el Laboratorio de Suelos de la Facultad de Agronomía de Azul (UNCPBA).

Las dataciones numéricas se obtuvieron por dos vías diferentes: a) fechados por ${ }^{14} \mathrm{C}$ (AMS) y b) dataciones por OCR (Oxidizable Carbon Ratio), método químico de reciente desarrollo para fechar materia orgánica de suelos y carbón (Frink, 1994 y 1995). Este método postula que compuestos como el carbón y los materiales húmicos son biológicamente reciclados en los suelos a una tasa lenta, pero mensurable, que progresa linealmente en relación con variables contextuales (temperatura media, precipitación media, textura media, profundidad, $\mathrm{pH}$ ) similares a las que intervienen en los procesos de formación de suelos (Frink 1995). Se modeliza entonces, a través de una fórmula, la relación entre estas variables y la tasa de oxidación del carbono orgánico, proporcionando una edad numérica. 


\section{Resultado}

En la localidad arqueológica Nutria Mansa el relevamiento geoarqueológico de las diferentes unidades del paisaje comprendió: 1. la planicie de inundación del valle fluvial (sitio NM1 y perfiles aledaños); 2 . la cercana lomada loéssica (divisoria) donde se ha registrado uno de los sitios en superficie (sitio NM1sup); y 3. una toposecuencia, en la que se efectuaron sondeos y barrenados desde la planicie de inundación hacia las divisorias a fin de obtener una visión horizontal de la dinámica geoambiental y sus correlatos arqueológicos a lo largo de una transecta perpendicular al cauce.

\section{Planicie de inundación del arroyo}

Estratigrafía del sitio Nutria Mansa 1 (NM1) (38²4' 54,2" S y 58 15' 50,1" O)

La estratigrafía general del sitio NM1 corresponde a depósitos fluviales y fluviolacustres referibles a los miembros Guerrero y Río Salado de la Formación Luján (Fidalgo et al. 1973). En NM1 se excavaron cinco cuadrículas de $2 \times 2 \mathrm{~m}$ y dos testigos (superficie total: $23 \mathrm{~m}^{2}$ ) y se levantaron seis perfiles estratigráficos a fin de evaluar las unidades representadas y sus variaciones laterales (Figuras 2 y 3). Los análisis texturales incluyeron el fraccionamiento de las arenas, que mostró un predominio de la arena muy fina (70 a 90\% de esta fracción).

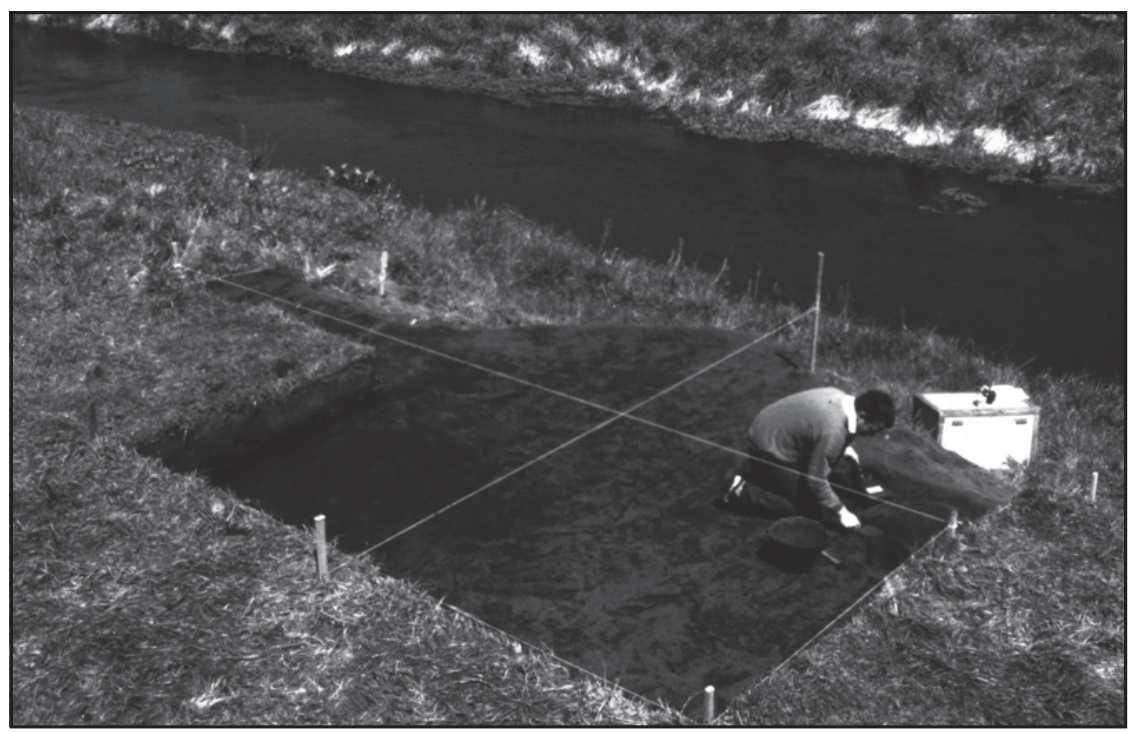

Figura 2. Vista de las cuadrículas

Se describe a continuación la estratigrafía de los perfiles 3, 5 y 6 con las profundidades de las unidades tomadas a partir de la superficie del terreno, ubicada a distintas medidas por debajo del nivel 0 de la excavación arqueológica ${ }^{1}$ (Figura 4). Estos perfiles ilustran la estratigrafía de sitio NM1. No se han registrado variaciones de importancia en las unidades representadas en cada perfil, sólo que manifiestan un ligero buzamiento hacia el NO. 
Perfil comparativo de la estratigrafía de NM1 ( $38^{\circ} 24^{\prime} 51,4^{\prime \prime}$ S y $58^{\circ} 15^{\prime} 50,6^{\prime \prime}$ O)

Como perfil comparativo de la estratigrafía del locus NM1 se utilizó la del primer sondeo efectuado en la localidad (sondeo 1), ubicado a unos $80 \mathrm{~m}$ aguas arriba del sitio, ya que es muy escasa la exposición de perfiles naturales en el área. En este sondeo se habían recuperado restos óseos $(n=89)$ y tres lascas de cuarcita de grano fino $(n=2)$ y basalto $(n=1)$. Los restos faunísticos que pudieron ser determinados a nivel específico pertenecen a Lama guanicoe con número mínimo de individuos igual a uno. La distribución vertical de los mismos comprendía unos $25 \mathrm{~cm}$ (desde los 0,40 a los 0,65 $\mathrm{m}$ de profundidad de la superficie del terreno).

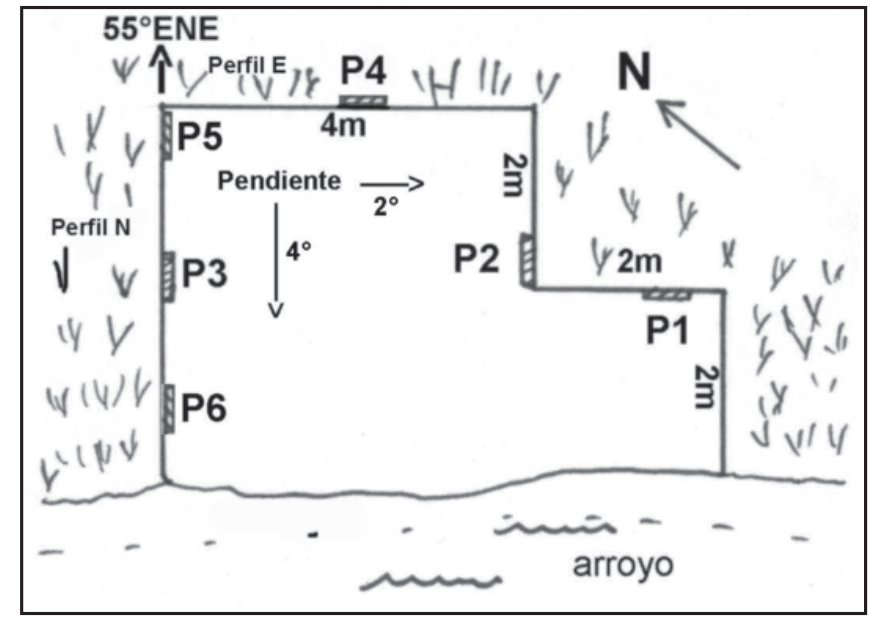

Figura 3 Planta general

La parte superior de esta secuencia estratigráfica se halla erosionada, estando ausente la unidad I reconocida en los perfiles de las cuadrículas excavadas en NM1 (Figura 5). La estratigrafía se inicia de esta manera con aquellos sedimentos finos referibles a la unidad II, con $60 \mathrm{~cm}$ de espesor. En ésta se distinguen dos suelos palustres (horizontes A-C y Ab1-Cb1)

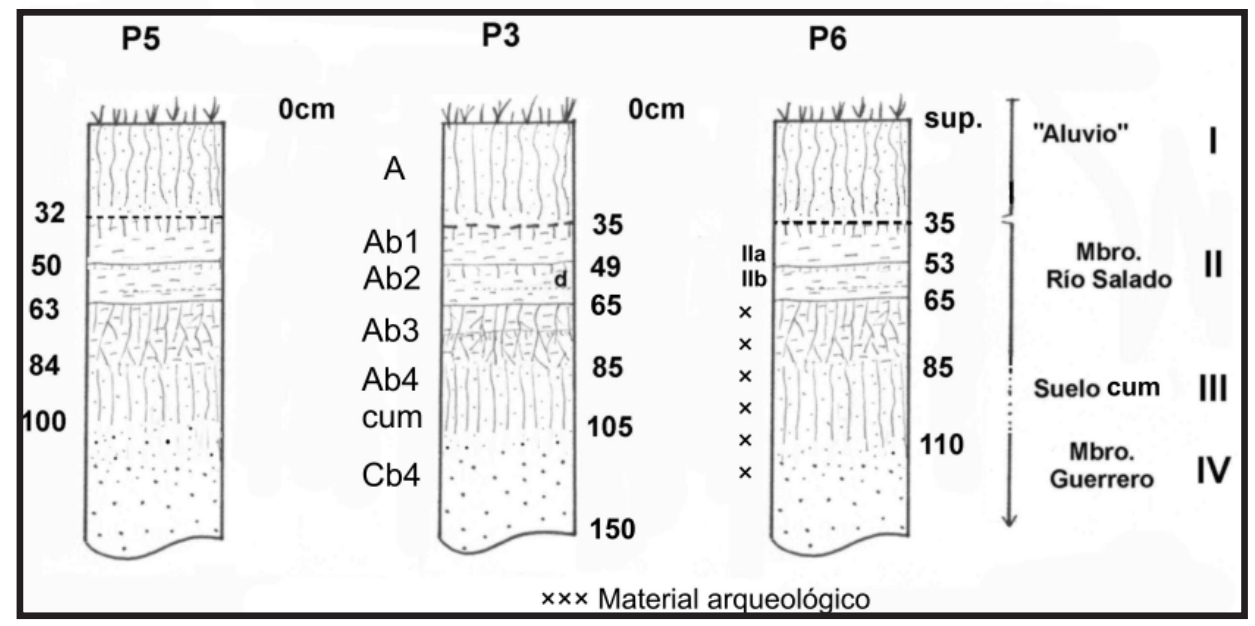

Figura 4 Perfiles estratigráficos 


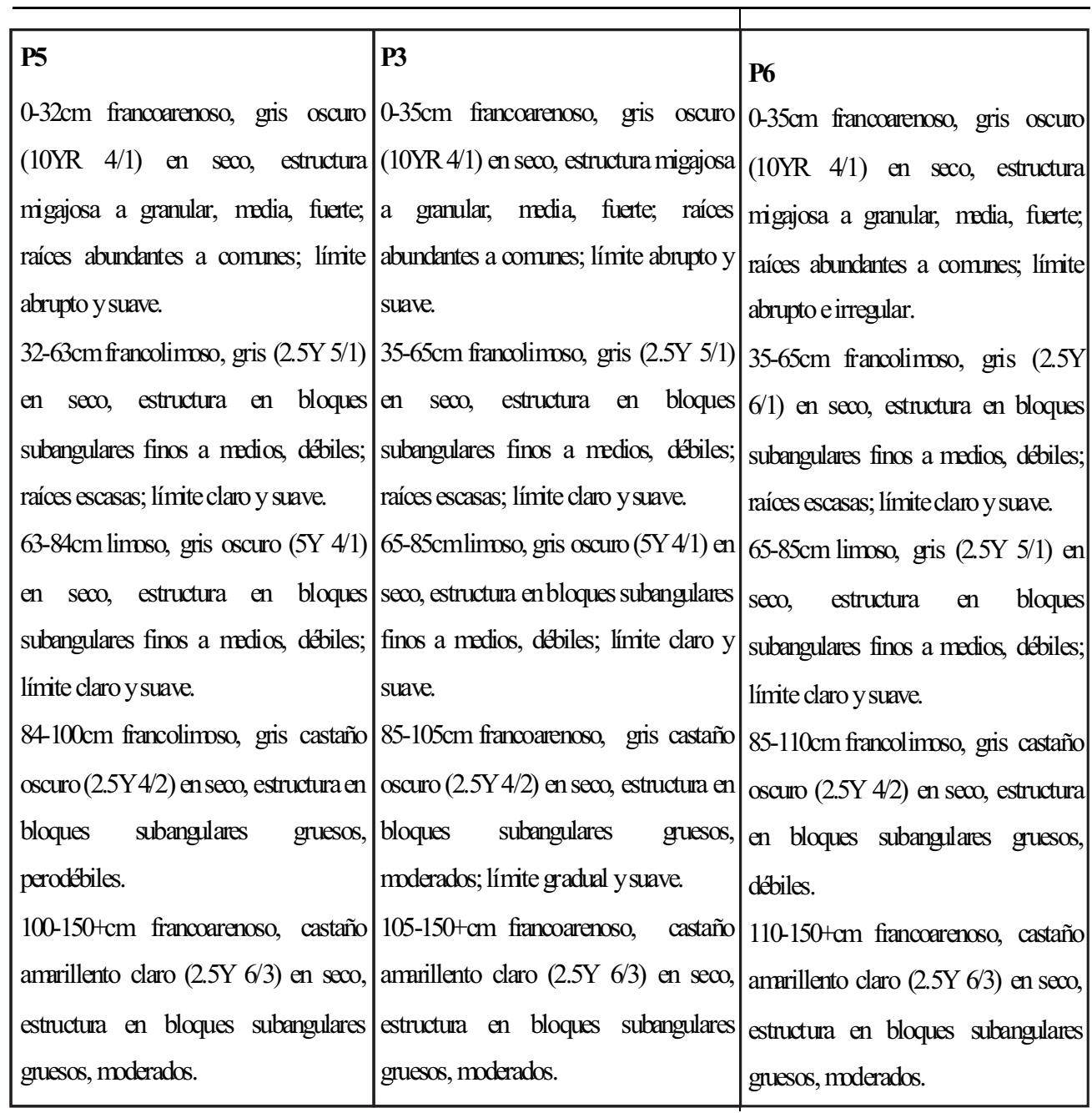

separados por un banco de diatomita semejante a aquél registrado en los perfiles relevados en NM1, pero algo más potente. Los materiales arqueológicos se hallaron por debajo de este banco asociados a la unidad II, en el horizonte A cumúlico que inicia la unidad III, a partir de unos $60 \mathrm{~cm}$ de la superficie. Este horizonte alcanza 1,60 m de profundidad, donde comienzan los típicos depósitos del Mbro. Guerrero de la Fm. Luján, que en este sector desarrollan halos de tinción alrededor de los canalículos de raíces.

Desde el punto de vista litológico se distinguen cuatro unidades principales, de arriba hacia abajo:

- unidad I (UE I; “aluvio"): depósito francolimoso que presenta rasgos pedológicos (humificación, bioturbación, presencia de raíces) en forma homogénea. Posee un contacto basal claramente discordante respecto a la unidad infrayacente que podría corresponder 
tanto a un contacto erosivo (a causa por ejemplo de un evento de inundación), como a perturbaciones de origen antrópico (remoción y mezcla de sedimentos por el arado).

- unidad II (UE II): depósito limoso que evidencia sedimentación de grano fino, con importante aporte bioclástico (diatomeas), ocasionalmente laminado. Corresponde a cuerpos de agua muy someros con presencia frecuente de suelos palustres y vegetación hidrófila. Estas facies son típicas del Holoceno medio e inicios del tardío en los valles fluviales y referibles al "Platense" o Mbro. Río Salado de la Fm. Luján de la literatura geológica.

- unidad III (UE III): es algo más arenosa, se halla constituida por un suelo oscuro, bien drenado, de tipo cumúlico. Se trata de un suelo de planicie de inundación que alterna baja sedimentación durante las crecientes, con pedogénesis en los momentos de estabilidad entre los pulsos de acreción. Ello permite el desarrollo de un suelo que crece verticalmente en forma pausada en la llanura aluvial (horizonte A cumúlico) y que mantiene vegetación abundante. Esta unidad es el contexto de hallazgo de la mayoría de la evidencia arqueológica en el sitio NM1 (Componente Inferior). Como la unidad anterior sería referible al "Platense" aunque no representa sus facies típicas. El acotado horizonte transicional AC y el C se observan desarrollados a expensas del "Lujanense" o Mbro. Guerrero de la Fm. Luján (unidad IV).

- unidad IV (UE IV): depósito predominantemente arenoso, caracterizado por una dinámica fluvial de mayor energía, con estructuras mecánicas de corriente y sedimentación más activa. El aporte bioclástico es muy escaso. El desarrollo de esta unidad corresponde a condiciones áridas a escala regional características del Pleistoceno tardío, tratándose de los típicos depósitos del Miembro Guerrero de la Fm. Luján.

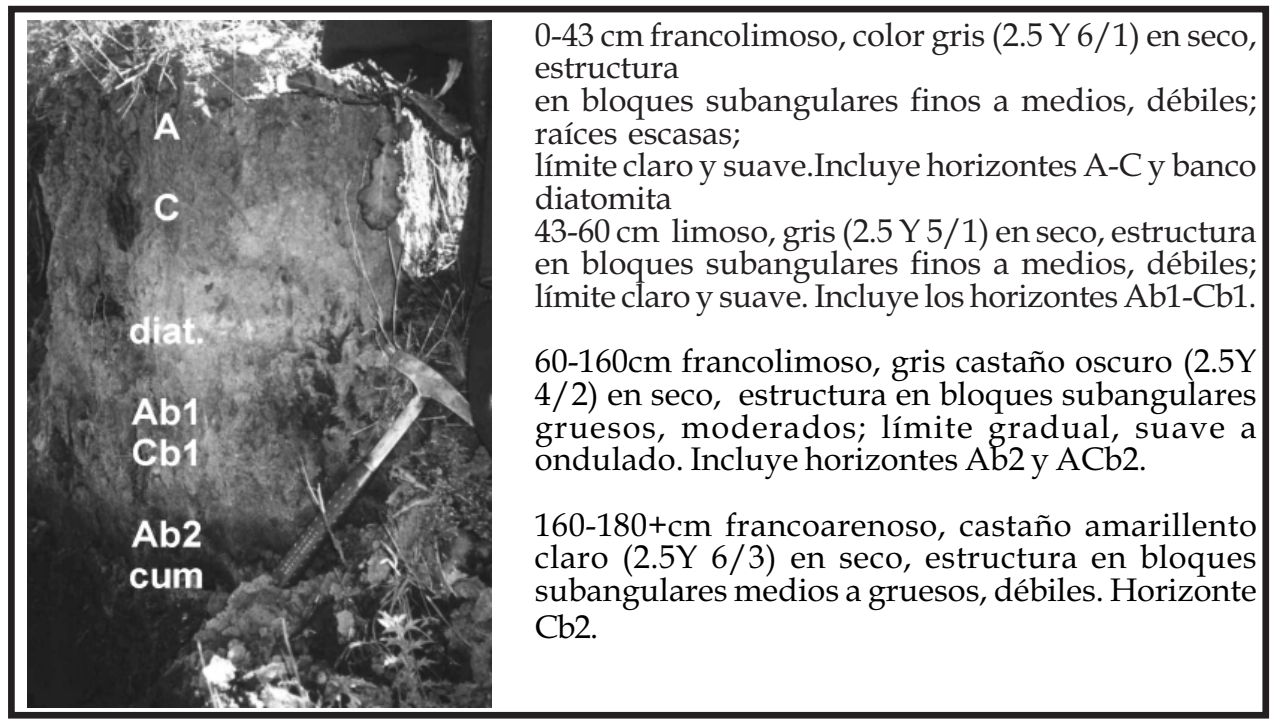

Figura 5. Interpretación de las unidades estratigráficas reconocidas en el arroyo Nutria Mansa 


\section{Condiciones geoquímicas en NM1}

Fueron evaluadas condiciones geoquímicas $(\mathrm{pH}, \mathrm{C}$ orgánico, salinidad, bases representadas) de los sedimentos de NM1 en vistas de examinar su incidencia en la preservación de los restos faunísticos recuperados en el sitio (Tabla 1).

\begin{tabular}{|l|l|l|l|l|lc|}
\hline Unidades & $\begin{array}{l}\% \quad \text { Carbono } \\
\text { orgánico }\end{array}$ & $\mathrm{pH}$ & Calcio* & Magnesio* & Sodio* & Potasio* \\
\hline I & 4.17 & 6,04 & 17,79 & 7,44 & 0,57 & 2,35 \\
2,46 \\
IIa & 2.59 & 8,07 & 22,28 & 10,31 & 1,86 & 1,82 \\
\hline IIb & 1.98 & 8,65 & 24,86 & 14,47 & 3,17 & 1,4 \\
\hline III & 1.34 & 8,89 & 14,80 & 17,16 & 3,98 & 3,24 \\
\hline IV & 0.09 & 8,68 & 8,91 & 15,06 & 1,02 \\
\hline
\end{tabular}

Tabla 1: Análisis geoquímicos de las distintas unidades estratigráficas en NM1

En NM1 se observa que el contenido salino en el conjunto de muestras no es elevado. La baja salinidad y la baja representación del Sodio apuntan a un ambiente continental para estos depósitos. Se destaca un incremento del Sodio en profundidad, con la misma tendencia que el $\mathrm{pH}$. El Magnesio se encuentra en valores absolutos y relativos altos, siguiendo también la tendencia del pH (mayor en la UE III). Es posible entonces que la alcalinidad que se observa en los valores de $\mathrm{pH}$ se halle vinculada al alto contenido de Magnesio, al que se suma la presencia de Sodio, aunque este último no presenta una concentración relativa que permita generar por sí mismo una alta alcalinidad.

Un ambiente muy alcalino crea condiciones desfavorables para la preservación de la fracción orgánica de los huesos, ya que facilita la hidrólisis del colágeno. Este mecanismo, junto a la probable acción de microorganismos, registrada en contextos semejantes (Gutiérrez 1998, 2001), contribuye a explicar la baja cantidad de colágeno presente en los tres restos óseos muestreados para su análisis. Los mismos presentaron una pérdida de peso por calcinación inferior a 2,7 \%, así como gran fragilidad en seco, con marcado astillamiento frente a la presión mecánica (Roberto Cordero com. pers. 2002). Así, la hidrólisis, con la subsiguiente pérdida de la fracción orgánica de los materiales faunísticos puede ser una de las causas que explican la alta proporción de fracturas en estado seco (81\%; véase Bonomo y Massigoge 2004) registradas en el sitio NM1.

\section{Cronología del sitio NM1}

Las dataciones del sitio NM1 fueron realizadas mediante dos métodos distintos, que arrojaron edades discordantes (Bonomo 2005). En primer lugar, se efectuaron fechados OCR de la materia orgánica contenida en los suelos, a partir de 6 muestras del perfil 4 . Las dataciones arrojaron las siguientes edades OCR: 467 años A.P. para la unidad 
estratigráfica I (UE I); 923 años A.P. y 1.837 años A.P. (UE IIa); 4.499 años A.P. (UE IIb); 5.970 años A.P. (UE III) y 7.630 años A.P. (transición entre UE III y IV).

Posteriormente, se efectuaron dataciones radiocarbónicas (AMS) sobre 3 muestras del Componente Inferior. Dado que los restos óseos presentaban un bajo contenido de colágeno se seleccionó para su procesamiento material dentario de Lama guanicoe bien preservado. A diferencia de las edades OCR, las edades $\mathrm{C}^{14}$ convencionales obtenidas para el Componente Inferior (UE IIb y III) fueron de $2.705+66$ años A.P., $3.080+110$ años A.P y $2.920+110$ años A.P., es decir sensiblemente menores.

Tres aspectos indican que las edades radiocarbónicas son las más representativas de la cronología de la ocupación humana del sitio NM1. Primero, la mayor confiabilidad del método AMS sobre el OCR que se encuentra aún en una etapa experimental. El cálculo de edades de tiempo de residencia media de la materia orgánica por el método OCR es muy dependiente de las variables contextuales y presenta limitaciones en suelos pobremente drenados (como los representados en esta planicie de inundación) debido a que la ecuación corresponde a un sistema dependiente de oxígeno (Frink 1995). En segundo lugar, puede esgrimirse la mejor calidad del material dentario procesado en comparación con la materia orgánica de los suelos, que es más sensible a verse afectada por perturbaciones. Finalmente, los fechados radiocarbónicos muestran edades muy similares entre sí, manifestando una elevada consistencia interna. Por lo tanto, privilegiando a las edades radiocarbónicas (2.700-3.100 años A.P.), se considera que las ocupaciones del Componente Inferior del sitio NM1 ocurrieron a inicios del Holoceno tardío.

\section{2-Divisorias (Lomadas Loessicas)}

El valle del arroyo Nutria Mansa se desarrolla entre suaves lomadas que representan los sectores más estables del paisaje. Están constituidas por loess (Fm. Pampiano sensu Fidalgo et al. 1973) y coronadas por suelos con un buen desarrollo de horizontes. Se trata por lo general de Argiudoles, es decir, suelos ricos en materia orgánica, bien drenados y que presentan un horizonte B rico en arcillas -Bt- (Figura 6). El desarrollo que poseen los suelos naturales en el área señala la existencia de superficies estabilizadas a lo largo de un extenso período, probablemente durante todo el Holoceno. Ello lleva a una concentración de la totalidad de la evidencia arqueológica en el horizonte A, incluyendo en ocasiones la parte superior del B (véanse Zárate et al. 2000-2002 y Favier Dubois y Politis 2006).

Por otro lado se observan en el sector numerosos suelos afectados por actividades agrícolas (Fig. 6B). En ellos el arado afectó los $40 \mathrm{~cm}$ superiores del suelo, lo que homogeneizó los horizontes A y la parte superior del horizonte Bt y formó un piso de arado en la zona inferior. Estos suelos disturbados corresponden a los sectores NM1sup y NM2sup, que evidencian la mezcla de diversos materiales prehispánicos con materiales modernos. 


\begin{tabular}{|c|c|}
\hline 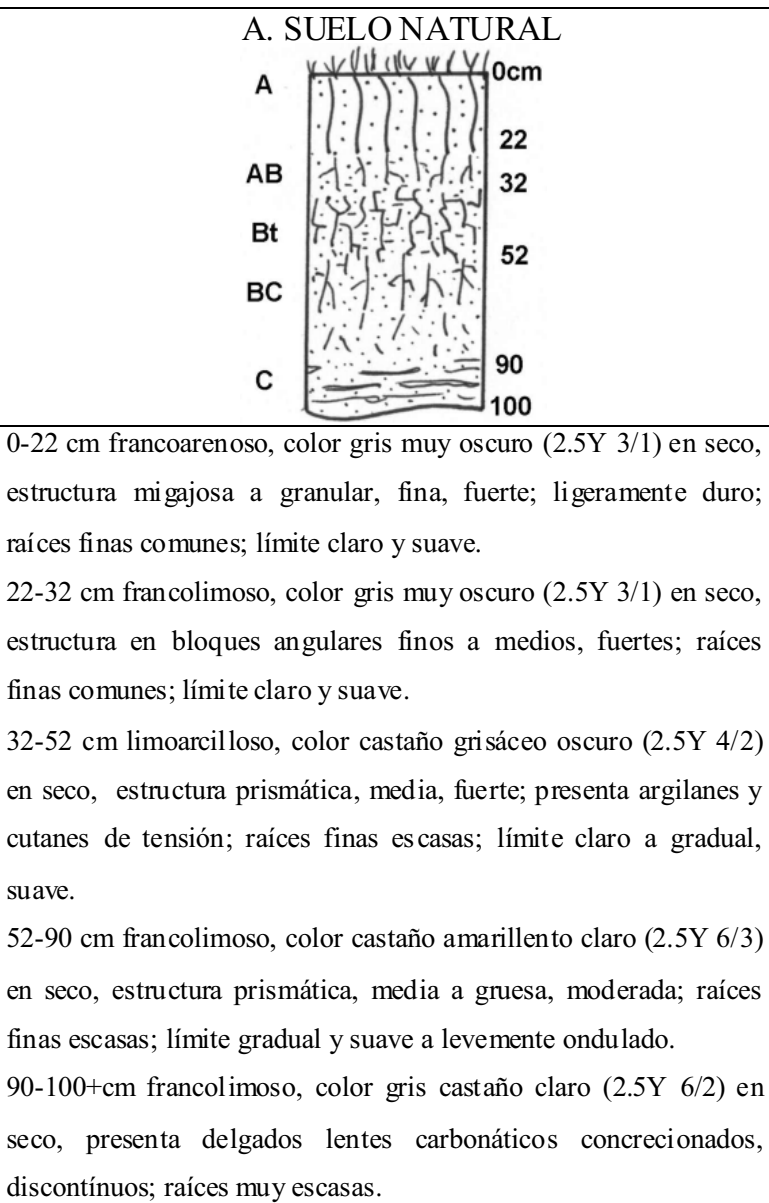 & $\begin{array}{l}\text { 0-38 cm francoarenoso; color gris } \\
\text { muy oscuro }(2.5 \mathrm{Y} 3 / 1) \text { en seco } \\
\text { masivo; blando; raíces finas comunes; } \\
\text { límite abrupto y suave. } \\
38-42 \mathrm{~cm} \text { arcilloso, color gris oscuro } \\
(2.5 \mathrm{Y} 4 / 1) \text { en seco, muy duro y } \\
\text { compacto (piso de arado). } \\
42-52+\mathrm{cm} \text { limoarcilloso, color castaño } \\
\text { grisáceo oscuro (2.5Y 4/2) en seco, } \\
\text { estructura prismática, media, fuerte; } \\
\text { presenta argilanes y cutanes de } \\
\text { tensión; raíces finas escasas. }\end{array}$ \\
\hline
\end{tabular}

\section{3- Toposecuencia}

Figura 6A y 6B.

A fin de evaluar los cambios en las unidades del paisaje en diferentes situaciones topográficas se realizaron 7 sondeos a lo largo de la pendiente desarrollada entre el valle fluvial (locus NM1) y el tope de la divisoria que se ubica hacia el NNE del mismo (suelo arado del perfil B). Esta pendiente, perpendicular al cauce, presenta una inclinación promedio de $4^{\circ}$. Se realizaron sondeos a lo largo de esta línea, partiendo del locus NM1, a 5, 10, 15, 20, 25 y 30 metros de las cuadrículas excavadas en este sitio (Figura 7). Se observa que los sedimentos fluviales, que alcanzan una potencia superior a los $3 \mathrm{~m}$ en las inmediaciones del cauce actual, se acuñan rápidamente en dirección a la suave lomada que limita el valle hacia el este, dando lugar a depósitos coluviales y eólicos.

Esta toposecuencia ilustra la variedad de contextos en la que puede encontrarse el registro arqueológico en una transecta perpendicular al cauce del arroyo que incluye las lomadas 


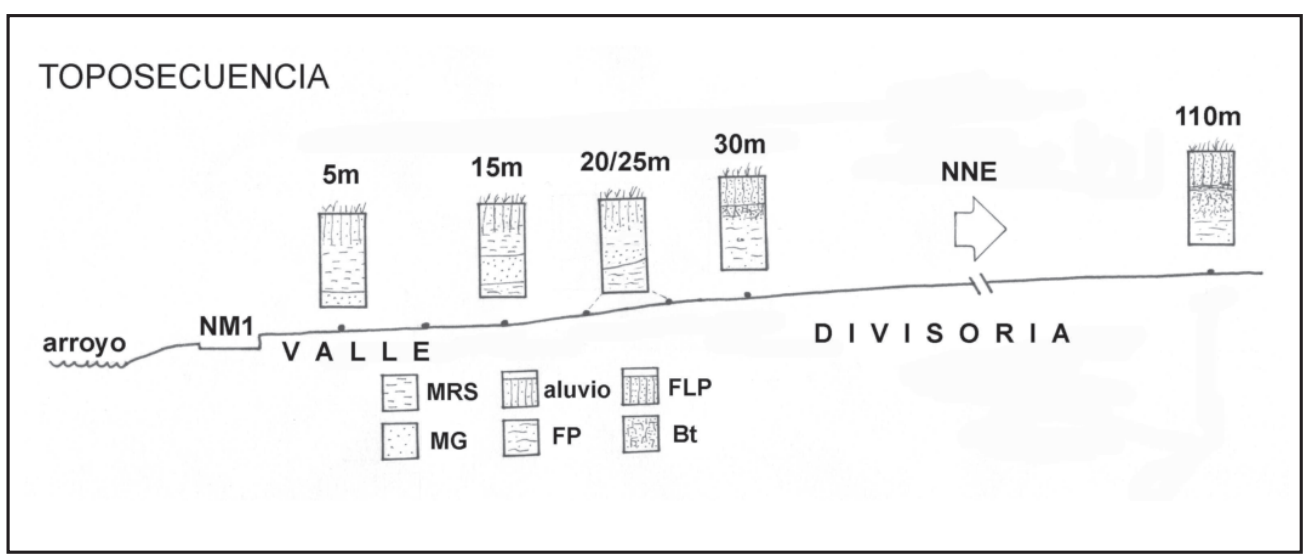

Figura 7. Toposecuencia

loéssicas, pendientes y el valle fluvial. Estas diferentes posiciones en el paisaje repercuten en la resolución, integridad y preservación potencial de ese registro (Tabla 2).

\section{Discusión y Conclusiones}

A partir de los estudios de microfósiles silíceos (fitolitos, frústulos de diatomeas, estomatocistos de crisófitas y espículas de espongiarios) efectuados en Nutria Mansa 1 (Bonomo et al. 2007) y del análisis geoarqueológico de su secuencia sedimentaria se observa que el contexto en el que se desarrollaron los eventos ocupacionales del Componente Inferior corresponde a ambientes fluviales y lacustres salobres con comunidades de gramíneas en sus márgenes. Los estudios de microfósiles silíceos indican que este ambiente de comienzos del Holoceno tardío se habría desarrollado bajo un clima templado, con un probable incremento posterior de la aridez y una estacionalidad marcada.

Con relación a los mamíferos registrados en este componente, se asocian tanto a condiciones áridas y frías como templadas y húmedas. En su mayoría provienen de un paleosuelo cuyas características, junto al registro areal de eventos pedológicos con edades similares (Tonni et al. 2001), indican condiciones cálidas y húmedas. Si bien existen algunas evidencias faunísticas y pedológicas que señalan un posible aumento de la temperatura con respecto a momentos anteriores, estas fluctuaciones no provocaron la retracción de animales propios de ambientes áridos y fríos. Por lo tanto, el contexto faunístico de NM1 estaría mostrando la convivencia de especies con diferentes requisitos ambientales, aspecto de suma importancia a la hora de evaluar la sensibilidad de determinados taxa como indicadores paleoclimáticos.

Dado que los materiales del Componente Inferior tienen una amplia distribución vertical (más de $80 \mathrm{~cm}$ ) y que en su gran mayoría se encuentran en un paleosuelo acrecional, es probable que representen distintos eventos de ocupación diacrónicos. Esto a su vez, se apoya en el análisis del material dentario de Lama guanicoe realizado por el Lic. C. Kaufmann (INCUAPA, UNCPBA) que indican varios episodios de caza entre noviembre y abril, no pudiéndose descartar la ocupación del sitio en otros meses del año (Bonomo et al. 2005). 
Los resultados de los estudios tafonómicos llevados a cabo en el sitio (Bonomo y Massigoge 2004; Bonomo et al. 2005) mostraron que los restos óseos presentan marcadas evidencias de meteorización y alteraciones diagenéticas que han influido en su preservación. En primer lugar, la covariación de las unidades anatómicas de Lama guanicoe con su densidad mineral ósea, señala que las partes esqueletarias menos densas están probablemente subrepresentadas en el conjunto. En segundo, lugar, el predominio estadios de meteorización 3 y 4 (sensu Behrensmeyer 1978) y los abundantes restos con evidencias de exfoliación indican que el material estuvo expuesto en la superficie del terreno durante un tiempo considerable. Respecto a esto último es necesario agregar que algunos (aunque escasos) especimenes poseen textura caliza y color blanquecino, lo cual señalaría que los mismos han sido afectados en forma intensa por la radiación solar (Mengoni Goñalons 1999). Esto concuerda con condiciones que alternan intervalos de estabilidad predominantes (pedogénesis) con sedimentación esporádica, como sugiere el desarrollo de la unidad III.

Si bien se registraron escasas evidencias de pisoteo en los niveles con mayor densidad de materiales arqueológicos, parte de los huesos largos y de los materiales líticos no se encontraron en posición horizontal (ángulos desde alrededor de $20^{\circ}$ a $90^{\circ}$ ). Entre los factores que pueden explicar esta disposición se encuentran el pisoteo sobre los sustratos blandos y plásticos del sitio y/o las características irregulares de la superficie sobre la que se depositaron. En relación a ello es importante especificar que, salvo en algunos casos, durante la excavación del sitio cuando se llegaba a los sedimentos del Miembro Guerrero (UE IV) dejaban de hallarse restos arqueológicos. La gran mayoría de los elementos que aparecían asociados a la unidad IV en realidad se encuentran en lentes negros del paleosuelo suprayacente (UE III). Esto está mostrando lo irregular del contacto entre el suelo (UE III) y el Miembro Guerrero (UE IV), hecho que resulta consistente con el pisoteo en presencia de sedimentos saturados de agua y cubiertos de vegetación.

Además de la detección de raíces actuales afectando los elementos óseos enterrados, en los huesos se observaron marcas de raíces lo cual también concuerda con la incorporación de los materiales en un paleosuelo. La acción de carnívoros y roedores a través del registro de marcas no fue observada en la muestra analizada. Aun así, no se descarta a estos animales y a la acción del agua como potenciales modificadores de la distribución espacial de los restos. Los resultados de los remontajes de artefactos líticos y de las uniones bilaterales de materiales dentarios de guanaco muestran desplazamientos horizontales de 8 a $250 \mathrm{~cm} \mathrm{y}$ verticales de 2 a $41 \mathrm{~cm}$ de ítems que se distribuyen entre los 84 y los $155 \mathrm{~cm}$ desde el nivel 0 . Estas migraciones verticales de magnitud posiblemente estén vinculadas con procesos tafonómicos vinculados con la plasticidad de los sedimentos portadores en presencia de pisoteo y con la acción continua de raíces.

En las superficies óseas también se observan manchas de manganeso (Mn) y hoyos de disolución química generados por el intercambio químico entre el hueso y su medio circundante ${ }^{2}$ (Johnson et al. 1997). Además, hay elementos con abrasión sedimentaria cuyos bordes y fracturas están redondeados y sus superficies presentan el desarrollo de pulidos o brillos por fricción. Las distintas superficies de estos huesos están diferencialmente abradidas evidenciando que han sufrido el impacto abrasivo de sedimentos in situ por el contacto constante con agua superficial (véase Gutiérrez 1998). La sumatoria de estos datos muestra que los materiales faunísticos y líticos pudieron estar al menos parte del tiempo en contacto con el agua de escorrentía. Esto se correlaciona con las condiciones ambientales inferidas 
para el desarrollo del paleosuelo, emplazado en una planicie de inundación, y con que éste último sea de tipo cumúlico, lo que implica el aporte de pulsos fluviales.

Por alguna razón, las características del sector debieron ser muy favorables para los grupos cazadores recolectores que ocuparon esta porción del paisaje fluvial, ya que la han visitado recurrentemente a lo largo del tiempo. Estas cualidades particulares no son evidentes en la actualidad, pero sin duda han influido en el uso redundante de este espacio en el pasado, que llevó a la acumulación de miles de ítems de evidencia arqueológica.

De acuerdo a las expectativas generadas a lo largo de la toposecuencia es probable que los conjuntos NM1sup y NM2sup, hallados en grandes superficies aradas sobre lomadas loéssicas, representen palimpsestos producidos por la mezcla horizontal y vertical de materiales procedentes de distintas ocupaciones. Junto a los objetos arqueológicos, se han registrado asimismo elementos recientes como loza, vidrio, ladrillo o plástico. La escasez de materiales óseos, que en general están muy degradados, se condice también con las inadecuadas condiciones de preservación inferidas para este contexto depositacional y contrasta con la gran cantidad de restos preservados en el valle fluvial.La presencia de cerámica en NM2sup permite sostener que por lo menos parte de sus ocupaciones corresponden al Holoceno tardío. Sin embargo, no sucede lo mismo con el locus NM1sup aun cuando está asociado espacialmente con el sitio estratigráfico NM1 correspondiente al Holoceno tardío. Las diferencias en cuanto a resolución vertical de los sedimentos depositados en el valle respecto a los de las divisorias (registradas a través de la toposecuencia) no permiten una correlación temporal entre ambos contextos. Como hemos visto, la zona de disturbación del arado afecta unos $40 \mathrm{~cm}$ de profundidad, erodando el horizonte $\mathrm{Bt}, \mathrm{y}$ alcanzando en algunas áreas la tosca de la Fm. Pampiano que se encuentra a poca profundidad del suelo actual. Esto se traduce en que el arado puede remover gran parte $o$ todo el paquete sedimentario correspondiente al Pleistoceno tardío-Holoceno, así como la evidencia que alberga.

En suma, los estudios geoarqueológicos forman parte de una perspectiva holística o contextual (Butzer 1982) del pasado cultural, que pone énfasis en el papel que el paisaje y la dinámica geoambiental juegan en la conformación del registro arqueológico y tafonómico. De esta manera, en el sitio NM1 el estudio de las unidades estratigráficas permitió evaluar el ambiente representado por la unidad portadora de los materiales líticos y óseos y los procesos de formación involucrados, caracterizando asimismo las condiciones geoquímicas de preservación de los abundantes restos faunísticos. Por otro lado, a escala del paisaje, la toposecuencia proporcionó una rápida visión de la variedad de contextos en los que puede quedar incluida la evidencia arqueológica a lo largo de una transecta perpendicular a un cauce fluvial. Las distintas subunidades representadas imprimieron propiedades espaciales y temporales diferenciales al registro arqueológico, que pueden ser vistas en términos de resolución, integridad y preservación de esos conjuntos. Ello ha permitido contextualizar la evidencia arqueológica recuperada en los loci NM1sup y NM2sup, en los que las actividades agrícolas podrían estar exponiendo la mayoría o la totalidad del registro arqueológico correspondiente a las ocupaciones humanas en la región. 


\section{Agradecimientos}

Los trabajos arqueológicos de campo y laboratorio se financiaron con fondos de dos proyectos denominados "Una perspectiva suprarregional de la arqueología del sudeste de la región pampeana” (UNLP 11/N503) y “Arqueología de las poblaciones indígenas del sudeste de la región pampeana desde un abordaje suprarregional" (PIP-CONICET 5424), dirigidos por Gustavo Politis. Asimismo el primer autor agradece al CONICET el Subsidio de Inicio de Carrera que financió gastos de los estudios geoarqueológicos.

\section{Notas}

${ }^{1}$ El nivel 0 se ubica en el perfil 3 a $13 \mathrm{~cm}$, en el perfil 5 a $12 \mathrm{~cm}$ y en el perfil 6 a $14 \mathrm{~cm}$ por encima de la superficie del terreno.

${ }^{2}$ Esto también pudo haber sido potenciado por el ambiente alcalino que rodeaba a los huesos en el depósito arqueológico.

\section{Bibliografia}

Aldazabal, V., N.E. Weiler y E. Eugenio

2004. Una perspectiva geoarqueológica para comprender la ocupación humana en la costa central de la provincia de Buenos Aires. Intersecciones en Antropología 5: 29-38.

Ameghino, F.

1880. La antigüedad del hombre en el Plata. Masson, París, Buenos Aires.

Behrensmeyer, A.K.

1978. Taphonomic and ecologic information from bone weathering. Paleobiology 4 (2): 150162.

Bonomo, $\mathrm{M}$.

2005 Costeando las llanuras. Arqueología del litoral maritimo pampeano. Sociedad Argentina de Antropología. Colección Tesis Doctorales. Buenos Aires.

Bonomo, M. y A. Massigoge

2004. Análisis tafonómico del conjunto faunístico del sitio arqueológico Nutria Mansa 1 (Pdo. de Gral. Alvarado). En Aproximaciones contemporáneas a la arqueología pampeana. Perspectivas teóricas, metodológicas, analíticas y casos de estudio, Martínez, G., M. Gutiérrez, R. Curtoni, M. Berón y P. Madrid (eds.), pp. 93-111. Olavaria, Facultad de Ciencias Sociales, UNCPBA.

Bonomo, M., G. Gómez y C. Kaufmann

2005. Análisis de los materiales faunísticos del Componente Inferior del sitio arqueológico Nutria Mansa 1 (pdo. de Gral. Alvarado, Pcia. de Buenos Aires, Argentina). Abstracts 2nd Internacional Meeting TAPHOS 05/4 Reunión de Tafonomía y Fosilización, Barcelona.

Bonomo, M., A. Zucol, B. Gutiérrez Téllez, A. Coradeghini y M.S. Vigna

2007. Palaeoenvironmental reconstruction in south-eastern Pampean Plains (Argentina) during Late Holocene based upon the analysis of siliceous microremains and mammal records of Nutria Mansa archaeological site. MS. 
Bórmida, $\mathrm{M}$.

1960. Investigaciones paletnológicas en la región de Bolívar (Pcia. de Buenos Aires). Anales de la Comisión de Investigaciones Científicas de la Provincia de Buenos Aires 1: 190-283.

Butzer, K.

1982. Archaeology as Human Ecology. Method and Theory for a Contextual Approach. Cambridge University Press.

Favier Dubois, C. M.

2006. Dinamica fluvial, paleoambientes y ocupaciones humanas en la localidad arqueológica Paso Otero, río Quequen Grande, Pcia. de Buenos Aires. Intersecciones en Antropología 7: 109127, Olavarría.

Favier Dubois, C. M. y M. Bonomo

2002. Evolución del paisaje, expectativas arqueológicas y procesos de formación en la Localidad Nutria Mansa (Pdos. de Gral. Alvarado y Lobería, Pcia. de Buenos Aires). Resúmenes del $3^{\text {er }}$ Congreso de Arqueología de la Región Pampeana Argentina: 76-77. Olavarría.

Fidalgo, F.; De Francesco, F. y Colado, U. 1973. Geología Superficial de las Hojas Castelli, J.M. Cobo y Monasterio (Argentina). Relaciones del 6to. Congreso Geológico Argentino. 103-138. Bahía Blanca.

Fidalgo, F., E. Tonni y J. Zetti

1971. Algunas observaciones estratigráficas en la laguna Blanca Grande (Pdo. de Olavarría, Pcia. de Buenos Aires). Etnia 14: 1-4.

Fidalgo, F., L. Meo Guzmán, G. Polits, M. Salemme, E. Tonni, J. Carbonari, G. Gómez, R. Huarte y A. Figini

1986. Investigaciones arqueológicas en el sitio 2 de Arroyo Seco (Pdo. de Tres Arroyos- Pcia. de Buenos Aires- República Argentina). En New Evidence for the Pleistocene Peopeling of the Americas, A. L. Bryan (ed.), pp. 221-269. Center for the Study of Early Man, University of Maine, Orono.

Flegenheimer, N., M. Zárate y M. Miguel

1999. El área de canteras Arroyo Diamante, Barker, Sierras de Tandil. Actas del XII Congreso Nacional de Arqueología Argentina III: 134-138. La Plata.

Flegenheimer, N. y M. Zárate

1993. The archaeological record in Pampean loess deposits. Quaternary International 17: 95100.

Frenguelli, J. y F. Outes

1924. Posición estratigráfica y antigüedad relativa de los restos de industria humana hallados en Miramar. Physis 7: 277-398.

González, M.I.

2005. Arqueología de alfareros, cazadores y pescadores pampeanos. Sociedad Argentina de Antropología. Colección Tesis Doctorales. Buenos Aires. 
González, M.A. y N.E. Weiler 1987/ 88. Sitio arqueológico Fortín Necochea. Informe geológico preliminar. Paleoetnológica 6: 55-63.

González de Bonaveri, M.I. y M.A. Zárate

1993-94. Dinámica de suelos y registro arqueológico: La Guillerma, provincia de Buenos Aires. Relaciones de la Sociedad Argentina de Antropología 19: 285-306.

Gutiérrez, M.A.

1998. Taphonomic effects and state of preservation of the guanaco (Lama guanicoe) bone bed from Paso Otero 1 (Buenos Aires Province, Argentina). Tesis de Maestría inédita, Texas Tech University, Texas.

Hrdlièka, A. (ed.)

1912. Early Man in South America. Smithsonian Institute, Bureau of American Ethnology 52, Washington.

Holliday, V. T., C. Reid Ferring y P. Goldberg

1993. The scale of soil investigations in archaeology. Effects of Scale on Archaeological and Geoscientific Perspectives, Editado por Julie K. Stein y Angela R. Linse. Geological Society of America, Special Paper 238.

Holliday, V. T., G. Martínez, E. Johnsony B. Buchanan 2003. Geoarchaeology of Paso Otero 5 (Pampas of Argentina). En Ancient evidences for paleo south americans: from where the south winnds blow, L. Miotti, M. Salemme y N. Flegenheimer (eds.), pp. 37-43. Center for the Studies of the First Americansand Texas A\& M University Press.

Johnson, E., M. Gutiérrez, G. Politis, G. Martínez y W. Hartwell

1997. Holocene Taphonomy at Paso Otero 1 on the Eastern Pampas of Argentina. En Proceedings of the 1993 Bone modification Conference, Hot Spring, South Dakota, L. Hannus, L. Rossum y R. Winham (eds.), pp. 105-121. Occasional Publication 1, Sioux Fall, Archaeology Laboratory, Augustana College.

Madrazo, G.

1972. Arqueología de Lobería y Salliquelo (Provincia de Buenos Aires). Etnía, 15: 1-18.

1979. Los cazadores a larga distancia de la Región Pampeana. Prehistoria Bonaerense: 12-67.

Martínez, G.A. y M.L. Osterrieth

2001. Estratigrafía, procesos formadores y paleoambientes. En: Cueva Tixi: cazadores y recolectores de las sierras de Tandilia Oriental. 1 Geología, Paleontología y Zooarqueología, D. Mazzanti y C. Quintana (eds.), pp. 19-34. Laboratorio de Arqueología, UNMdP, Publicación Especial 1, Mar del Plata.

Menghín, O. y M. Bórmida

1950. Investigaciones prehistóricas en las cuevas de Tandilia. Runa 3: 5-36. 
Mengoni Goñalons, G. L.

1999. Cazadores de guanacos de la estepa patagónica. Sociedad Argentina de Antropología, Colección Tesis Doctorales, Buenos Aires.

Messineo, P., P. Barros, D. Poire y L. Gómez Peral

2004. Características Litológicas de los Niveles de Chert o Ftanitas en las Sierras Bayas (Partido de Olavarría, Provincia de Buenos Aires. En: Aproximaciones Contemporáneas a la Arqueología Pampeana. Perspectivas Teóricas, Metodológicas, Analíticas y Casos de Estudio, editado por G. Martínez, M. A. Gutierrez, R. Curtoni, M. Berón y P. Madrid, pp. 305-317. Olavarría.

Osterieth, M., G. Martínez, D. Zurro, A. Zucol, M. Brea y D. Mazzanti

2002. Procesos de formación del Sitio 2 de la Localidad Arqueológica Amalia: evolución paleoambiental. En Del Mar a los Salitrales. Diez mil Años de Historia Pampeana en el Umbral del Tercer Milenio, D. Mazzanti, M. Berón y F. Oliva (eds.), pp. 343-354. Facultad de Humanidades, UNMdP, SAA, Mar del Plata.

Pérez Meroni, M. y A. Blasi

1997. Sitio arqueológico "El Ancla" provincia de Buenos Aires. Ensayos y experimentación de sedimentos pelíticos locales para la manufactura carámica. En Arqueología Pampeana en la década de los '90, M. Berón y G. Politis (eds.), pp.175-185. Museo de Historia Natural de San Rafael/INCUAPA, UNICEN, Olavarría.

Politis, G.

1984. Arqueología del Area Interserrana Bonaerense. Tesis Doctoral inédita, Facultad de Ciencias Naturales y Museo, UNLP, La Plata.

Tapia, A.

1937. Las cavernas Ojo de Agua y Las Hachas. Boletín de la Dirección de Minas y Geología, 43: $1-126$.

Teruggi, M.E.

1968. Geología y sedimentología de las cuevas de la Cuchilla de las Aguilas (Sierras de Tandil, Prov. de Buenos Aires). Etnía 7: 13-20

Tonni, E.P., A.L. Cione y A.J. Figini

2001. Chronology of Holocene Pedogenetic Events in the Pampean Area of Argentina. Current Research in the Pleistocene, 18: 124-127.

Zárate, M. y N. Flegenheimer

1991. Geoarchaeology of the Cerro La China Locality (Buenos Aires, Argentina): Site 2 and Site 3. Geoarchaeology: An International Journal, 6 (3): 273-294.

Zárate, M. y A. R. Prieto

1997. Role of the Geological Sciences in the archaeological research of Buenos Aires Province, Argentina. Quaternary of South America and Antartic Peninsula 10 (1994): 311-320.

Zarate, M.; M. I. González de Bonaveri; N. Flegenheimer y C. Bayón

2000-2002 Sitios arqueológicos someros: el concepto de sitio en estratigrafía y sitio de superficie. Cuadernos del Instituto Nacional de Antropología y pensamiento Latinoamericano (INAPL) 19: 635-653, Buenos Aires. 
Zavala, C, S. Grill, D Martínez, H. Ortíz y R. González

1992. Análisis Paleoambiental de depósitos cuaternarios. Sitio Paleoicnológico Monte Hermoso I, Provincia de Buenos Aires. Actas de las Terceras Jornadas Geológicas Bonaerenses: 3137. La Plata.

Zetti, J. E.P. Tonni y F. Fidalgo

1972. Algunos rasgos de la geología superficial en las cabeceras del arroyo Azul (Provincia de Buenos Aires). Etnía 15: 28-34. 